\title{
VENTAJA COMPETITIVA DE LA CADENA DE SUMINISTROS: ALIANZAS, ASIMETRÍAS ORGANIZATIVAS Y CONFLICTOS
}

\author{
MARIANA ZERÓN FÉLIX \\ Facultad de Comercio y Administración Victoria \\ Universidad Autónoma de Tamaulipas México \\ zfmariana@uat.edu.mx \\ GUILLERMO MENDOZA CAVAZOS \\ Facultad de Comercio y Administración Victoria \\ Universidad Autónoma de Tamaulipas México \\ gcavazos@uat.edu.mx \\ JUANA MARÍA QUEVEDO DÍAZ \\ Facultad de Comercio y Administración Victoria \\ Universidad Autónoma de Tamaulipas México \\ jquevedo@uat.edu.mx
}

\section{RESUMEN}

El presente estudio teórico pretende analizar la integración de acuerdos de colaboración dentro de la cadena de suministros, lo cual va permitir unir una firma a su cliente, a los proveedores y a otros miembros de una cadena de suministros que brindan servicios robusteciéndola con la intención de satisfacer al cliente final; lo anterior con el fin de lograr la pauta de formalizar alianzas estratégicas. Se presenta un análisis descriptivo de teorías que fundamentan la determinación de las estrategias de las organizaciones en función de la ventaja competitiva, se propone que los socios comerciales deben identificar sus asimetrías administrativas y el conflicto que puedan surgir entre ellos para proponer las discrepancias como oportunidades y realizar los ajustes necesarios en la administración de la alianza, estableciendo canales que permitan un alto grado de comunicación eficiente entra las partes.

PALABRAS CLAVE: CADENA DE SUMINISTROS, ALIANZAS ESTRATÉGICAS, ASIMETRÍAS ORGANIZATIVAS, CONFLICTO.

\section{ABSTRACT}

The present theoretical study analyzes integration partnerships within the supply chain which allow a firm to bring clients, suppliers and other members of the supply chain together, to provide services by strengthening the supply chain with the intention of formalizing strategic alliances, and to finally satisfy customers. Through an analysis of theories that describe how organizations determine their own strategies based on their competitive advantages, it is suggested that trading partners should identify managerial asymmetries and conflicts that may arise between them, they should observe discrepancies as opportunities in order to make the necessary adjustments in their management of alliances, and establish, with higher degrees of efficient, communication between parts.

KEYWORDS: SUPPLY CHAIN, STRATEGIC ALLIANCE, ORGANIZATIONAL ASYMMETRIES, CONFLICT.

\section{INTRODUCCIÓN}

Actualmente, empresas de producción, distribución y comercialización integran alianzas de manera estratégica con el objeto de constituir aso- 
ciaciones que van más allá de lo referente a los acuerdos de logística, como se han observado en forma tradicional. Los nuevos acuerdos se formalizan y operacionalizan con la intención de permitir un flujo consistente de bienes e información desde los proveedores de materias primas hasta el consumidor final.

El interés en el tipo de asociaciones mencionado es el incrementar la ventaja competitiva de cada uno de los integrantes en la cadena de suministros (Simental, 2005). De tal manera que se vuelve indispensable identificar aquellas coincidencias y diferencias entre empresas que forman una cadena, las cuales intervienen en el mejor desempeño, tanto de manera individual como en la cadena completa.

\section{INTEGRACIÓN DE CADENAS DE SUMINISTROS}

En presencia de la tendencia del proceso de globalización de la industria, la competencia en los mercados no es a través de dos empresas, actualmente la competencia entre un proveedor y un proveedor competitivo es su relación proveedor - comprador ( Wu, Lin, Chien y Hung, 2011).

Mientras que las presiones de la competencia empujan a cortar costos, las empresas han descubierto que la noción de la cadena de suministros adiciona un valor a las organizaciones donde negocia para realizar una ventaja competitiva (Ellington, 1998). Así se establecen acuerdos donde se expresa el compartir recursos tecnológicos, físicos y financieros, con el objetivo de que cada eslabón de la cadena de suministros cumpla con su función de manera eficiente, logrando mayores rendimientos para cada uno de ellos.
En este proceso de integración en donde un consumidor y un proveedor forman una fuerte extensión social, económica, de servicio, y de ciertas uniones técnicas en un tiempo determinado, por el intento de reducir costos totales así como de aumentar valor, se pretende alcanzar una ventaja competitiva mutua (Ellington, 1998).

El agregar valor a un producto o servicio a lo largo de la cadena de suministros es esencial para la competitividad de las organizaciones y en algunas ocasiones vital para la supervivencia de las mismas. Otros autores (Mentzer et al., 2001; Turban, 2002) definen la cadena de suministros como un grupo de tres o más entidades (organizaciones o individuos) directamente envueltos en flujo ascendente y descendente de productos, servicios, finanzas e información desde su origen (materias primas) hasta el cliente (usuario final). A la definición es indispensable abstraer la importancia de la integración, de la comunicación y de la coordinación entre las funciones y la organización, lo cual creará valor para el cliente (Gillyard, 2003).

La cadena de suministros es la integración de entidades en sus actividades y la coordinación de las mismas, con el objetivo global de satisfacer a sus clientes con base en la ventaja competitiva creada ante la asociación. Por lo tanto, para comprender el sistema de la cadena de suministros es necesario coordinar los elementos que forman la administración de la misma.

La coordinación de la infraestructura, de los procesos y del conocimiento generado permite a las entidades establecer estrategias de negocios que los lleve a fortalecer su ventaja competitiva; dichas estrategias se presentan a través de la formación de alianzas estratégicas y permiten otorgarle un carácter formalista a la asociación de los entes integrantes y al flujo del conocimiento surgido por cada eslabón de la cadena. 


\section{Ventaja competitiva: su relación con la alianza estratégica}

Para la década de los noventa, las tendencias comenzaron a inclinarse hacia las relaciones más cercanas con los clientes y en las alianzas estratégicas con proveedores; es entonces cuando compañías de un amplio espectro de industrias enfocan sus estrategias competitivas para aprovechar las competencias y capacidades innovadoras encontradas en las redes inter-organizacionales formadas por clientes y proveedores de una misma cadena de suministros (Simental, 2005).

Lo anterior provoca el estudio de la utilización de la estrategia de negocios de formación de alianzas, con la intención de lograr un mejor desempeño de los procesos del producto o servicios de las funciones de cada eslabón de una cadena de suministros.

Las empresas buscan obtener mayores rendimientos para cumplir con el compromiso de la administración de maximizar el valor de la inversión de cada uno de los socios, es así que la misma administración busca el uso óptimo de los recursos para aprovechar aquellos aspectos que los hace únicos y mejores ante sus competidores.

La ventaja competitiva de las compañías de clase mundial depende primordialmente de las inversiones estratégicas en las capacidades del producto y los procesos. A corto plazo, estas inversiones restringen los márgenes de ganancia, pero son esenciales para la creación de capacidades innovadoras que generarán prosperidad continua (Ponce, 2003) y lograrán penetración en el mercado. Por tanto, las alianzas estratégicas son asociaciones multifacéticas de largo plazo que suceden entre dos o más organizaciones orientadas hacia un objetivo bien definido, en las que ambas partes comparten riesgos y beneficios (Simental, 2005); su formación permite a las empresas asociarse con aquellas empresas con recursos que complementen los propios para alcanzar una ventaja competitiva que les permita enfrentar el ambiente cambiante de los negocios (Wu, Lin, Chien y Hung, 2011)

La competencia desempeña un papel vital en la determinación de la posición estratégica de una organización. Cuando una empresa tiene muchos competidores, sus posiciones estratégicas están en riesgo. Por lo que en una situación altamente competitiva una entidad económica hace frente a su competencia tan bien como su producto logre los retos de diferenciarse. Por lo tanto, se define expresamente la ventaja competitiva que va a destacar a la cadena de suministros formada. Es también indispensable identificar el nivel de asociación que existe dentro

TABLA 1

\section{TIPOLOGÍA DE ASOCIACIÓN}

\begin{tabular}{|c|l|}
\hline TIPO DE ASOCIACIÓN & \multicolumn{1}{c|}{ DESCRIPCIÓN } \\
\hline Tipo I & $\begin{array}{l}\text { Se lleva a cabo la coordinación de las actividades y la planificación sobre una base limitada y entre ciertas funciones de } \\
\text { diferentes empresas. }\end{array}$ \\
\hline Tipo Il & Se extienden más allá de la coordinación básica de las actividades. \\
\hline Tipo III & $\begin{array}{l}\text { Es evidente un importante nivel de integración operativa; asociaciones que requieren amplia comunicación y colaboración } \\
\text { entre organizaciones. }\end{array}$ \\
\hline
\end{tabular}

Fuente: Lambert al. (1996). 
de una alianza estratégica, Lambert et al. (1996) definen tres niveles de asociación (Tabla 1).

En la tabla 1, se describe los tipos de asociación que según Lambert, et al. (1996) pueden existir entre las empresas, con el fin de que se identifiquen y establezcan acuerdos sobre la relación que existirá entre sus actividades.

Para el mismo investigador, el nivel de asociación se basa en un conjunto de factores complejos e interdependientes (culturales, de organización, de estilo de dirección y liderazgo, de procesos de control y de comunicación). Dichos factores son importantes porque influyen en uno o más determinantes de las relaciones inter-organizacionales.

Las asociaciones pueden desarrollarse a partir de una necesidad (introducción a nuevos mercados, recursos tecnológicos, financieros, generación de investigación y desarrollo, etcétera). El potencial para ejercer poder o control sobre cualquier otra organización o sus recursos determina el grado de asimetría en algunas relaciones. Los socios comerciales pueden corresponderse unos con otros en cuanto a cooperación, colaboración y coordinación.

De esta forma varias empresas pueden explotar distintos recursos y conocimiento tecnológico en favor de incrementar la ventaja competitiva del sistema completo de la cadena de suministros; por lo tanto, una organización puede buscar a compañías dispuestas a cooperar dentro de la línea ascendente o descendente de una cadena.

La integración de recursos entre empresas aumentan la eficiencia de al menos una de las empresas asociadas, proporciona estabilidad o capacidad de responder a la incertidumbre del ambiente, así como la legitimidad sobre las actividades de al menos uno de los socios; así el nivel de los factores enumerados influyen en la fuerza de la integración (Wu et al., 2011).
Al definir el tipo de acuerdo de asociación que habrá entre los integrantes de la cadena de suministros, debe existir un proceso bien estipulado de planificación y control de la estructura de trabajo, de la estructura de la organización, del flujo del producto en la estructura instalada, de la estructura del producto, de los métodos de gestión, de la estructura de poder y liderazgo y de la cultura y la actitud (Cooper, et al., 1997; Lambert et al., 1998; Lambert y Cooper, 2000). Así como de la función de la mediación del mercado para alinear responsablemente la oferta de productos y servicios de la empresa con las demandas de los clientes (Fisher, 1997).

\section{Creación de las ventajas competitivas}

Al integrarse la cadena de suministros en acuerdos de asociación, colaboración o coordinación mediante la formación de alianzas estratégicas, son identificadas las características que permiten penetrar en un mercado, por las cuales el cliente final prefiera un producto o servicio ante otros. Por tanto se requiere el control de los costos, los tiempos de entrega y la calidad de los procesos internos de producción; aparte del objetivo de logar la satisfacción del cliente, por lo que los proveedores de línea ascendente y descendente necesitan al menos cubrir estos tres factores (Wu et al., 2011), los cuales son derivativas de las dimensiones competitivas.

Por otra parte, las dimensiones son: precio o costo, calidad, velocidad de entrega, confiabilidad de entrega, afrontar los cambios de la demanda, flexibilidad y velocidad para la introducción de nuevos producto, entre otros aspectos (Chase et al., 2009; Boyer y Lewis, 2002).

- Costo o precio: se refiere al logro de mantener costos bajos en el proceso productivo, ya que en toda industria existe un segmento de mercado que suele comprar a bajo costo. Para competir con éxito en este nicho, la empresa debe ser un productor que ofrece 
costos bajos, a pesar de que esto no siempre garantice la rentabilidad ni el éxito; se debe asegurar que el producto llegue en la cadena de suministros al cliente final a un costo mínimo (Chase et al., 2009; Bilbao, 2003; Wu et al., 2011).

- Calidad: una alta calidad representa lograr un alto estándar de que el producto o servicio sea aceptado por el cliente; la cual debe desarrollarse en el momento del diseño del producto o servicio y durante el proceso de implementación, según sea el caso. Por lo tanto, la meta de la calidad del proceso es producir bienes y servicios que no tengan defectos; varios autores confirman la dimensión de la calidad como elemento primordial en la definición de estrategia de operaciones en lo concerniente en la relación entre socios comerciales de una cadena de suministros (Heimeriks y Schreiner, 2002; Boyer y Lewis, 2002; Liongrokagart, 2001).

- Velocidad y confiabilidad en la entrega: la capacidad de la empresa para entregar su producto con mayor rapidez (en la fecha y en la cantidad pactada) que sus competidores es fundamental; lo cual otorga a la cadena de suministros un elemento primordial de integración para su estrategia de operaciones (Liongrokagart, 2001; Boyer y Lewis, 2002).

- Afrontar los cambios de la demanda: la capacidad de la compañía para responder ante incrementos o disminuciones de la demanda es muy importante para su capacidad competitiva. Una compañía que afronta un incremento de la demanda suele incurrir en algunos errores, cuando la demanda es fuerte y se incrementa, los costos van disminuyendo de forma continua en razón de las economías de escala y no es difícil justificar las inversiones en nuevas tecnologías. Pero cuando la demanda disminuye, la reducción podría requerir muchas decisiones difíciles, como despedir a empleados y otras reducciones de activos. La capacidad para manejar debidamente la demanda dinámica del mercado a largo plazo es un elemento esencial de la estrategia de operaciones (Chase, et al., 2009).

- Flexibilidad y velocidad para la introducción de nuevos productos: la flexibilidad desde una perspectiva estratégica se refiere a la capacidad de una compañía para ofrecer una amplia variedad de productos a sus clientes; así como la de cambio ante las necesidades nacientes o crecientes del mercado mediante la transformación o desarrollo de un nuevo producto o servicio (Chase et al., 2009; Liongrokagart, 2001; Boyer y Lewis, 2002).

- Otros criterios específicos del producto: existen productos o servicios especiales que requieren atención en aspectos específicos como lo son: enlace y apoyo técnico, cumplimiento de la fecha de lanzamiento, apoyo del proveedor después de la venta y otras dimensiones (Chase et al., 2009).

Aunado a lo anterior, en un mundo en el que las organizaciones deben competir no solo con la disponibilidad, costo y calidad de sus productos y servicios, sino también con la calidad de la información que genera para ser utilizada por sus socios, las tecnologías de la información sincronizan la cadena de suministros a través del intercambio de información y procesos críticos de los negocios (Bilbao, 2003).

\section{Asimetría organizativa}

El éxito de la cadena de suministros al formar alianzas no se garantiza al tener una ventaja competitiva destacada, implica también un proceso de gestión que permita administrar las similitudes y diferencias presentes entre las em- 
presas que la conforman. Es indispensable así para el cumplimiento de los objetivos comunes, identificar las fronteras y las funciones frecuentes de los socios que forman la cadena (Kolp y Faulkner, 2007).

Las alianzas entre empresas con culturas, tamaño y experiencia en cooperación similares son más duraderas y estables. La similitud entre las culturas de las organizaciones, el volumen de activos de las mismas y el grado de experiencia en cooperación afecta positivamente el desempeño de la alianza (Harrigan, 1999; Heimeriks y Schreiner, 2002). Sin embrago, las asimetrías en el tipo de relación entre los socios no siempre corresponden a aspectos organizativos (Harrigan, 1999); sino que van surgiendo según las características de cada acuerdo de colaboración.

Al formar un acuerdo de colaboración los socios comerciales deben establecerlo dentro de un modelo básico de interdependencia basado en la confianza, lo cual fortalecerá su asociación (Wu et al., 2011).

Por otra parte, cada organización mantiene su propio estilo de dirección, lo cual difiere considerablemente entre empresas; destaca que los directivos mantengan en la toma de decisiones diferentes enfoques, más racionales o más intuitivos, así como en los sistemas de control y los procesos de comunicación (Escriba, 2002; Raham y Khorn, 2010; Méndez, 2007) o que actúen con horizontes temporales diferentes respecto a los plazos y las prioridades de cada empresa (Gibson y Cevich, 1994).

El éxito de las alianzas estratégicas se halla fuertemente ligado, entre otros muchos aspectos, a la elección del socio con el cual se coopera (Méndez, 2007). Los socios comerciales deben construir la confianza, la negociación y la cooperación para integrase dentro de un sistema que permita combinar de manera eficiente sus recursos (Wu et al., 2011).
Sin embargo, aunque las diferencias culturales han sido ampliamente tratadas en la literatura, son pocos los estudios que analizan con profundidad el conflicto relacionado con las diferencias organizativas y, por el momento, no ha sido posible lograr un consenso suficiente en relación con los efectos de las asimetrías organizativas sobre la puesta en marcha y el correcto desarrollo de una alianza (Escribá, 2002).

Si se apunta a una relación positiva entre la similitud de las empresas en términos organizativos y la existencia de un mayor nivel de confianza, la complementariedad de los recursos o capacidades aportados por cada socio no parecen tener una influencia tan clara (Johnson et al., 1996), así como tampoco el hecho de tener una comunicación constante y fluida entre los socios comerciales. Sin embargo, desde el punto de vista de alianzas estratégicas internacionales Brouthers et al., (2009) proponen que la selección de un socio debe incluir la consideración de las habilidades complementarias, la cultura corporativa, los objetivos compatibles y un conmensurado nivel de riegos.

En una alianza, la complejidad del acuerdo se define en función del número de socios, la diversidad de las actividades por realizar (por funciones, número de mercados o productos), la necesidad de interacción entre los socios y la naturaleza (más o menos rutinaria) de las tareas (Killing, 1998); es así que el logro de una simetría organizativa dentro de una alianza estratégica depende de la comunicación entre sus socios comerciales, lo cual facilita su éxito.

La participación en el diseño y la integración de la estrategia de operaciones y suministro debe ser un proceso facilitador y de identificación de las asimetrías administrativas, para ser abordadas en cuanto a su importancia e impacto en la formalización de los acuerdos de colaboración, con la intención de fortalecer la ventaja competitiva de la cadena completa. 


\section{Conflicto en la alianza}

En cualquier relación, bajo cualquier grado de interdependencia con otros socios comerciales, se requiere considerar el análisis de conflictos que se pueda suscitar. Para analizar este fenómeno entre organizaciones puede observarse como referencia aquellas problemáticas que surgen entre grupos que pertenecen a una organización; y así remitirse a estudios centrados en el conflicto entre grupos externos a las organizaciones para determinar las causas de este fenómeno en el ámbito empresarial (Escriba, 2002; Raham y Khorn, 2010; Méndez, 2007). El conflicto se produce cuando en la toma de decisiones conjunta existe una diferencia de objetivos o una diferencia en la percepción de la realidad (Escribá, 2002).

La necesidad sentida de la toma de decisiones conjunta se produce, fundamentalmente, como consecuencia de la interdependencia entre los grupos involucrados, aspecto inherente a los acuerdos de cooperación entre empresas (Escriba, 2002; Méndez, 2007).

Así bien, las alianzas se convierten en un escenario propenso a la aparición de conflictos como resultado de las diferencias en los objetivos particulares o en las percepciones de los participantes (Escribá y Menguzzato, 1999). Es así que la participación de cada uno de los socios comerciales en el diseño de la estrategia de operaciones debe proponer los ajustes necesarios para la alineación con sus estrategias particulares, lo que permite dar cumplimiento a sus objetivos y a los establecidos para la cadena de suministros.

Sin embargo, cabe destacar que no todos los acuerdos de cooperación presentan un mismo grado de interdependencia (Escribá, 2002). Siguiendo a Thompson (1967), existen tres tipos de interdependencia entre grupos.

1. Interdependencia común o mancomunada: Una alianza puede tener una división simple del trabajo, con unos ajustes mínimos que solo requieren que cada socio comparta información sobre el progreso de sus iniciativas para la consecución de los objetivos del acuerdo (Escribá, 2002).

2. Secuencial: La interdependencia va en la continuidad de los procesos de negocio, en el momento en que para realizar su función depende del cumplimiento anterior de la actividad de otro de los socios, por lo que el buen funcionamiento se relaciona con el accionar de los otros socios.

3. Recíproca: La interdependencia es mayor cuando resulta de la anticipación de una compleja y solapada división del trabajo que provoca ajustes continuos y mutuos, además de requerir que cada socio vincule sus actividades propias con las de los otros de un modo regular y próximo.

A mayor nivel de interdependencia aumenta la necesidad de tomar decisiones de un modo conjunto, por tanto un mayor riesgo a generar conflicto entre los socios. Además, se incrementa la exigencia de que la información sea transmitida de un modo claro y eficaz a aquellos que participan en dichas decisiones, por lo tanto, los costos de procesamiento de información también se incrementan (Escribá, 2002), y el tiempo dedicado a la gestión de la cadena de suministros puede traslaparse o entorpecer la gestión de la operaciones de cada socio.

De acuerdo con Kocabasoglu (2002) las tecnologías de información se han convertido en una herramienta fundamental en la gestión de la cadena de suministros al permitir que el departamento de compras lleve a cabo de manera eficiente varias tareas relacionadas con las adquisiciones, para darle agilidad a los procesos productivos y, de la misma manera, para generar procesos de comunicación más eficientes entre 
las organizaciones en relación con la interdependencia de sus actividades.

El grado de interdependencia en una alianza está condicionado a las tareas incluidas y a la división del trabajo establecida en el acuerdo (Gulati y Singh, 1998), lo cual podría de la misma manera afectar y propiciar la aparición de conflictos y moderar o acentuar los efectos de las asimetrías existentes entre los socios (Escribá, 2002).

Dada una necesidad de toma conjunta de decisiones, las fuentes de conflicto podrán derivarse a las diferencias de objetivos y percepciones entre los grupos u organizaciones que interactúan (March y Simon, 1958). De tal modo que se deben considerar elementos de moderación para el conflicto dentro de la estrategia de operaciones y suministro entre los socios, así como el posible impacto en su desempeño.

\section{CONCLUSIONES}

Con base en la revisión de la literatura, se propone que las organizaciones que conforman cadena de suministros y colaboran formalmente en alianzas estratégicas tengan la perspectiva de analizar sus asimetrías organizacionales para fortalecer su ventaja competitiva.

La propuesta se basa en la fuerza que se genera en las relaciones coordinadas de procesos completos de negocios entre varias empresas en su objetivo de llegar con un desempeño óptimo al consumidor final.

El definir una alianza como estrategia de negocio para la colaboración y la coordinación de las actividades como cadena de suministros permite que se establezcan las vías por las cuales los recursos fluirán; de acuerdo con las funciones que desempeñen permite enfocar una toma de decisiones conjunta en beneficio de cada uno de los socios.
Como primer paso se establece la identificación y definición clara de la ventaja competitiva con que enfrentarán a otras cadenas dentro del mercado, como segundo paso una planeación estratégica conjunta, es decir, que la alianza establezca en su acuerdo formal los elementos propios de una gestión; dicho proceso requiere de un estudio minucioso para alinear la planeación con el de cada organización integrante de la cadena.

Durante el segundo paso debe quedar establecido el tipo de interdependencia que se planteará, por lo tanto surgirán las primeras confrontaciones sobre las diferencias existentes en lo concerniente a cada cultura organizacional (sistemas de comunicación, dirección, control, entre otros).

En un tercer paso se propone evaluar las asimetrías organizativas, potenciales causas de conflictos; por lo cual la propuesta se basa en enfocar una etapa de la planeación en donde se establezcan los sistemas de comunicación de la cadena, las líneas de mando, los límites de funciones y de toma de decisión, así como los canales y procesos que consideren más aptos para la resolución de conflictos.

Al encaminar el proceso de toma de decisiones mediante sistemas ágiles y eficientes de información entre los socios comerciales, se irán así resolviendo los conflictos estratégicos y consecuentemente se brindará la oportunidad de solventar también los conflictos a nivel operativo.

No solamente las contradicciones son una fuente de tensiones entre los actores organizacionales, sino que también puede proporcionar la oportunidad para el surgimiento de un nuevo conocimiento organizacional de la alianza en sí. Así que otro factor primordial para una futura investigación sería el conocimiento generado en la integración de acuerdo de colaboración surgido de cadenas de suministros integradas en alianza estratégicas. 
Es importante observar que las contradicciones también varían en su origen, algunas influyen en la evolución de las relaciones entre organizaciones de una manera más sustancial que otras. Por lo cual se propone que los socios de una alianza identifiquen y desarrollen una cultura del conflicto, dando pie a establecer esquemas de gestión de las relaciones inter-organizacionales.

Para concluir, parafraseando a Simental (2005), el éxito de toda la estrategia de cadena de suministros es el intercambio adecuado de información entre sus integrantes, sin demeritar la selección adecuada del tipo de acuerdo de colaboración por establecer. Por tanto, el tipo de asociación es muy significativa en la integración de la cadena, su desarrollo necesita de confianza, compromiso y cooperación para alcanzar resultados altamente eficientes (Wu et al., 2011).

\section{Referencias}

Bilbao, F. I. (2003) Información, Tecnología y Cadena de Suministro. Boletines de Estudios Económicos, LVIII (179), $297-314$.

Boyer, K. y Lewis, M. (2002) Competitive Priorities: Investigating the need for TRADE-OFSS in Operations Strategy. Production and Operations Management, 11 (1), 9- 20.

Brouthers, L.E., Mukhopadhyay, S., Wilkinson, T.J., Brouthers, K.D. (2009). International market selection and subsidiary performance: A neural network approach. Journal of World Business, 44, 262-273

Cooper, M. C., Lambert, D. M. y Pagh, J. D. (1997). Supply Chain Management: More than a New Name for Logistics. The International Journal of Logistics Management, 8(1), 1 - 13.

Chase, R. B., Jacobs, F. R. y Aquilano. (2009). Administración de operaciones, producción y cadena de suministros. D.F.: McGraw Hill.

Elligton, B. (1998) Processes and the Evaluation of Trust in Interfirm Collaborative Relationships: A longitudinal Study. Tesis de Doctor en Filosofía. Universidad de Northwestern, Evanston, Illinois. Estados Unidos.
Escribá, A. (2002) Análisis de la influencia de las asimetrías organizativas, en el performance de las Alianzas entre empresas. Managment Interactivas, 6(3).

Escribá, A. y Menguzzato, M. (1999). Determinantes y Efectos del Comportamiento Cooperativo de los Socios en las Alianzas entre Empresas. Revista ICADE (Instituto Católico de Administración y Dirección de Empresas), 48, 25-43

Fisher, M.L. (1997). What is the Right Supply Chain for Your Product?. Harvard Business Review, (Mar-Abr), 105 116.

Gibson I., y Cevich D., (1994). Organizaciones. D.F., México: McGraw Hill.

Gillyard, A. E. (2003) The relationships among supply chain characteristics, logistics and manufacturing strategies, and performance. Tesis Doctoral. Universidad Estatal de Ohio, Estados Unidos.

Gulati, R. y Singh, H. (1998). The architecture of cooperation: managing coordination costs and appropriation concerns in strategic alliances. Administrative Science Quarterly, 43(4), 781-814.

Harrigan, K. R. (1999). Estimation of cross country differences in industry production functions. Journal International Economics, 47(2), 267 - 293.

Heimeriks KH. y Schreiner M. (2002). Relational quality and alliance capacity: A conceptual framework for their influence on alliance performance. SMS Strategic Management Society Annual International Conference, Septiembre, $22-25$,

Killing, P. (1998). Un día después de una Joint Venture. Harvard Deusto Business Review, 86, 42 - 46.

Kolp, M. y Faulkner, S. (2007). Patterns of Organizational Modeling. International Journal of Enterprise Information Systems, 3(3), 1 - 22.

Lambert, D. M., Emmelhainz, M. A. y Gardner, J.T. (1996). Developing and implementing Supply Chain Partherships. The International Journal of Logistics Management, 7(2), $1-17$.

Lambert, D. M., Cooper, M. C. y Pagh, J. D. (1998). Supply Chain Management: Implementation Issues and research Opportunities. The International Journal od Logistics Management, 9(2), 1 - 19.

Lambert, D. M. y Cooper, M. C. (2000). Issues in Supply Chain Management. Industrial Marketing Management, 29, 65 $-83$. 
Liongrokagart, J. (2001). Measuring and enhancing the performance of closely linked decision making units in supply chains using customer satisfaction data. Tesis de Doctor en Filosofía, Clemson University.

March, J. G. y Simon, H. A. (1958). Organizations. New York: John Wiley \& Sons.

Méndez, K. (2007). Relación de Cooperación entre Empresas de Alojamiento de Medio Rural a partir del Estudio de Factores Intangibles. Simposio Internacional de Turismo y Ocio. Barcelona: ESADE.

Mentzer, J.T., Dewitt, W., Keeber, J., Min, S., Nix, N., Smith, C. y Zacharia, Z. (2001). Defining Supply Chain Management. Journal of Business Logistics, 22(2), 1 - 25.

Ponce, J. I. (2003). Determinación de indicadores de desempeño de la Cadena Suministro según la Teoría de restricciones. Tesis para obtener el grado de maestro. Instituto Tecnológico y de Estudios Superiores de Monterrey, Monterrey, Nuevo León. México.

Raham, N. y Korn, HJ. (2010). Alliance structuring behavior:relative influence of alliance type and specific alliance experience. Management Decision, 48 (5), $809-825$.

Simental, C. E. (2005). Factores críticos para la administración de la cadena de suministros (SCM) en empresas del estado de Nuevo León. Tesis para obtener el grado de Maestro, Instituto Tecnológico y de Estudios Superiores de Monterrey, Monterrey, Nuevo León. México.

Thompson, J.D. (1967). Organizations in action. New York: McGraw-Hill.

Turban, E. (2002) Electronic Commerce; a Managerial Perspective. New Jersey: International Edition, Pearson Education.

Wu, M., Lin, Y., Chien, F., y Hung, Y. (2011). A Study on the Relationship among Supplier Capability, Parthership and Competitive Advantage in Taiwan's Semiconductor Industry. International Journal of electric Business Management, 9 (2), $122-138$.

Recibido: 21 de setiembre de 2012 Aceptado: 30 de enero de 2013 